\title{
A comparative study of microbial diversity and community structure in marine sediments using $\operatorname{poly}(\mathrm{A})$ tailing and reverse transcription-PCR
}

\author{
Tatsuhiko Hoshino ${ }^{1,2}$ * and Fumio Inagaki, ${ }^{1,2}$ \\ ${ }^{1}$ Geomicrobiology Group, Kochi Institute for Core Sample Research, Japan Agency for Marine-Earth Science and Technology, Nankoku, Kochi, Japan \\ ${ }^{2}$ Geobio-Engineering and Technology Group, Submarine Resources Research Project, Japan Agency for Marine-Earth Science and Technology, \\ Nankoku, Kochi, Japan
}

\section{Edited by:}

Anna-Louise Reysenbach, Portland

State University, USA

Reviewed by:

Gordon Webster, Cardiff University, UK

Jinjun Kan, Stroud Water Research Center, USA

${ }^{*}$ Correspondence:

Tatsuhiko Hoshino, Geomicrobiology Group, Kochi Institute for Core

Sample Research, Japan Agency for Marine-Earth Science and Technology, Monobe B200, Nankoku, Kochi 783-8502, Japan

e-mail: hoshinot@jamstec.go.jp
To obtain a better understanding of metabolically active microbial communities, we tested a molecular ecological approach using poly $(A)$ tailing of environmental $16 \mathrm{~S} r R N A$, followed by full-length complementary DNA (cDNA) synthesis and sequencing to eliminate potential biases caused by mismatching of polymerase chain reaction (PCR) primer sequences. The RNA pool tested was extracted from marine sediments of the Yonaguni Knoll IV hydrothermal field in the southern Okinawa Trough. The sequences obtained using the poly(A) tailing method were compared statistically and phylogenetically with those obtained using conventional reverse transcription-PCR (RT-PCR) with published domain-specific primers. Both methods indicated that Deltaproteobacteria are predominant in sediment $(>85 \%$ of the total sequence read). The poly $(A)$ tailing method indicated that Desulfobacterales were the predominant Deltaproteobacteria, while most of the sequences in libraries constructed using RT-PCR were derived from Desulfuromonadales. This discrepancy may have been due to low coverage of Desulfobacterales by the primers used. A comparison of library diversity indices indicated that the poly $(A)$ tailing method retrieves more phylogenetically diverse sequences from the environment. The four archaeal 16S rRNA sequences that were obtained using the poly(A) tailing method formed deeply branching lineages that were related to Candidatus "Parvarchaeum" and the ancient archaeal group. These results clearly demonstrate that poly $(A)$ tailing followed by cDNA sequencing is a powerful and less biased molecular ecological approach for the study of metabolically active microbial communities.

Keywords: 16S rRNA, less biased diversity analysis, hydrothermal sediment

\section{INTRODUCTION}

Numerous studies on natural microbial communities from a variety of environments have been undertaken using 16S rRNA gene sequencing mediated by polymerase chain reaction (PCR) with oligonucleotide primers. In the past decade, high-throughput next-generation sequencing (NGS) technologies have facilitated the identification of a diverse array of organisms that are rare in terms of biomass and could not be examined using previous molecular assays such as Sanger sequencing analysis of clone libraries (Sogin et al., 2006; Webster et al., 2010). Despite the fact that the latest NGS technologies enable reading only of relatively short sequence fragments $(\sim 500 \mathrm{bp})$, these so-called "deep sequencing" methods are still powerful tools that ultimately may enable researchers to obtain a more holistic understanding of microbial communities in their natural environments (Fuhrman, 2009). Considering the current limitations of NGS technology, full-length $16 \mathrm{~S}$ rRNA gene sequences are better suited to downstream analytical methods such as fluorescence in situ hybridization.

The original designs of most of the PCR primers used for the analysis of $16 \mathrm{~S}$ rRNA genes were based on known sequences deposited in public databases. Researchers have since cautioned that these primer sequences contain mismatches with respect to environmental 16S rRNA genes (Baker et al., 2003, 2006; Teske and Sørensen, 2008), which may lead to considerable bias in interpreting results (Hong et al., 2009). In addition, primer sequence mismatches may have a negative impact on the amplification efficiency of PCR analyses (Acinas et al., 2005; Sipos et al., 2007; Bru et al., 2008). Regardless of the presence of sequence mismatches, the use of PCR with primers may introduce biases associated with the next base adjacent to annealed oligonucleotide primers (Ben-Dov et al., 2012).

One way to avoid these bias issues is to employ PCRindependent metagenomic approaches. For example, a complete 16S rRNA gene sequence can be obtained by analyzing the sequences of genomes or large genome fragments, providing taxonomic information along with information on other functional genes. However, metagenomic approaches may not be well-suited to focused studies of microbial diversity and community structure that involve a large number of samples. In fact, it has been reported that only a small portion of inserts in fosmid libraries contain $16 \mathrm{~S}$ rRNA genes (Vergin et al., 1998; Takami et al., 2012). 
Another method that can avoid the possibility of bias caused by primer mismatching is the addition of a poly(A) tail to the $3^{\prime}$ end of fractionated 16S rRNA prior to synthesis of the fulllength complementary DNA (cDNA; Botero et al., 2005). Since the technique does not involve the use of published primers, we anticipate that this method will enable recovery of full-length environmental 16S rRNAs, potentially illuminating as yet unknown microbial community constituents that have otherwise been difficult or impossible to detect using conventional PCR-dependent molecular approaches (Inagaki et al., 2002). In the present study, we tested this hypothesis using poly $(\mathrm{A})$ tailing of full-length $16 \mathrm{~S}$ rRNA and reverse transcription-PCR (RT-PCR) with domainspecific primers, and compared sequence libraries prepared from a marine sediment sample collected from the Yonaguni Knoll IV hydrothermal field.

\section{MATERIALS AND METHODS}

\section{SAMPLING OF MARINE SEDIMENTS}

The sediment samples used in this study were obtained from the Yonaguni Knoll IV hydrothermal field in the southern Okinawa Trough $\left(24^{\circ} 50.544^{\prime} \mathrm{N}, 122^{\circ} 42.878^{\prime} \mathrm{E}\right.$, water depth: $\left.1,371 \mathrm{~m}\right)$ using a push corer, and were collected during the JAMSTEC NT10-06 cruise involving $R V$ Natsushima and ROV Hyper-Dolphin (Dive \#1111, April 17, 2010). Sediment samples were anaerobically placed in autoclaved 250-ml glass bottles using a nitrogen flush and the bottles were sealed with a rubber cap and stored at $4^{\circ} \mathrm{C}$ until analysis.

\section{RNA EXTRACTION AND PURIFICATION}

Bulk environmental RNA was extracted from $8 \mathrm{~g}$ of sediment using an RNA PowerSoil ${ }^{\circledR}$ Total RNA Isolation Kit (MO BIO Laboratories, Inc., Solana Beach, CA, USA) according to the manufacturer's instructions. The extracted RNA was electrophoresed on a $2 \%$ agarose gel for $30 \mathrm{~min}$ in $1 \times$ TAE [Tris-acetateethylenediaminetetraacetic acid (EDTA)] buffer, and the gel was stained with $1 \times$ SYBR Green II (Life Technologies Japan, Tokyo, Japan) to visualize $16 \mathrm{~S}$ and $23 \mathrm{~S}$ rRNA. The rRNA was recovered from the gel using a Recochip (Takara Bio, Japan), and then further purified using a PureLink RNA Mini Kit (Life Technologies Japan) according to the manufacturer's instructions. The quality of the recovered 16S rRNA was verified by electrophoresis using an automated capillary electrophoresis system (Experion; BioRad Laboratories, Tokyo, Japan) and an Experion RNA HighSens Analysis Kit.

\section{POLY(A) TAILING AND COMPLEMENTARY DNA SYNTHESIS}

We compared two molecular approaches [poly(A) tailing and RTPCR] for examining metabolically active microbial communities. The approaches are summarized in Figure 1. Since the reaction buffer composition and source of poly(A) polymerase can reportedly affect the efficiency of the poly(A) tailing reaction (Raynal and Carpousis, 1999; Sillero etal., 2001), we used two commercially available poly(A) polymerases: Escherichia coli poly(A) polymerase (New England BioLabs, hereafter denoted as NEB) and Takara Bio poly(A) polymerase. Each poly(A) tailing reaction was conducted in $20 \mu \mathrm{l}$ of reaction mixture containing $10 \mu \mathrm{l}$ of purified 16S rRNA solution. The other components of the reaction mixture were as follows: for NEB polymerase, $1 \times$ reaction buffer (50 mM Tris- $\mathrm{HCl}, 250 \mathrm{mM} \mathrm{NaCl}$, and $10 \mathrm{mM} \mathrm{MgCl}_{2}$ ), $1 \mathrm{mM}$ ATP, and $0.25 \mathrm{U} / \mu \mathrm{l}$ of poly(A) polymerase; for Takara Bio, $1 \times$ reaction buffer [ $50 \mathrm{mM}$ Tris- $\mathrm{HCl}, 10 \mathrm{mM} \mathrm{MgCl}_{2}, 2.5 \mathrm{mM} \mathrm{MnCl}_{2}$, $250 \mathrm{mM} \mathrm{NaCl}$, and $1 \mathrm{mM}$ dithiothreitol (DTT)], $0.5 \mathrm{mg} / \mathrm{ml}$ of bovine serum albumin, $0.5 \mathrm{mM}$ ATP, and $0.1 \mathrm{U} / \mu \mathrm{l}$ of poly $(\mathrm{A})$ polymerase. After incubation at $37^{\circ} \mathrm{C}$ for $30 \mathrm{~min}$, the reaction was stopped by adding $2 \mu \mathrm{l}$ of $250 \mathrm{mM}$ EDTA. The poly(A)-tailed 16S rRNA was subsequently purified using a NucleoSpin ${ }^{\circledR}$ RNA XS Kit (Takara Bio). The cDNA of full-length 16S rRNA was synthesized and amplified by PCR using a SMARTer ${ }^{\text {TM }}$ Pico PCR cDNA Synthesis Kit (Takara Bio) according to the manufacturer's instructions.

\section{REVERSE TRANSCRIPTION-PCR}

Reverse transcription-PCR was performed to obtain nearly fulllength rRNA gene sequences from the purified 16S rRNA without poly(A) tailing using a One-Step PrimeScript RT-PCR Kit (Takara Bio). The bacterial domain-specific primers 26F (AGAGTTTGATCCTGGCTCA; Hicks etal., 1992) and 1492R (GGYTACCTTGTTACGACTT; Loy et al., 2002) were used for RTPCR. The reaction mixture consisted of $1 \times$ PrimeScript buffer, $300 \mathrm{nM}$ of each primer, $0.8 \mu \mathrm{l}$ of PrimeScript Enzyme mix, $1 \mu \mathrm{l}$ of $16 \mathrm{~S}$ rRNA (diluted 1,000-fold), and water to $20 \mu \mathrm{l}$. First, reverse transcription was performed at $50^{\circ} \mathrm{C}$ for $30 \mathrm{~min}$ followed by inactivation of the reverse transcriptase at $94^{\circ} \mathrm{C}$ for $2.5 \mathrm{~min}$. Next, synthesized cDNA was amplified by PCR under the following condition: 20 cycles of $94^{\circ} \mathrm{C}$ for $30 \mathrm{~s}, 54^{\circ} \mathrm{C}$ for $30 \mathrm{~s}$, and $72^{\circ} \mathrm{C}$ for $90 \mathrm{~s}$. The number of PCR cycles used in this study was determined by selecting a cycle number in the log-linear phase of the realtime PCR amplification curve (i.e., before the plateau phase). The PCR products were purified using NucleoSpin Extract II Columns (Takara Bio) and stored at $-20^{\circ} \mathrm{C}$ until further analysis.

\section{CLONING AND SEOUENCING}

The PCR products obtained using poly(A) tailing and RT-PCR were cloned into the $\mathrm{pCR}{ }^{\circledR} 2.1-\mathrm{TOPO}^{\circledR}$ vector and transformed into competent E. coli DH5 $\alpha$ (Life Technologies Japan, Tokyo, Japan). For RT-PCR, the cloned inserts were sequenced using an ABI 3130xl genetic analyzer (Life Technologies Japan) with the primers M13M4, M13rev, 926R/F (Liu et al., 1997), and 1390R (Zheng et al., 1996). For the poly(A) tailing method, sequencing was first performed using the M13 primers followed by screening of the $16 \mathrm{~S}$ rRNA sequence using a hidden Markov model implemented in version 3.0 of the HMMER software package (Eddy, 1998), as described elsewhere (Lagesen et al., 2007; Huang et al., 2009). The screened 16S rRNA inserts were sequenced using primers 338R/F (Amann et al., 1990), 515R/F (Walters et al., 2011), 926R/F, and 1390R to assemble full-length 16S rRNA sequences. A primer walking approach was employed for inserts that could not be sequenced using the primers described above. The sequences were trimmed and assembled to obtain consensus sequences using Sequencher software (Hitachi Software, Tokyo, Japan). Chimeric sequences were removed using the UCHIME program (Edgar et al., 2011) implemented in the Mothur Utility package (Schloss et al., 2009). 


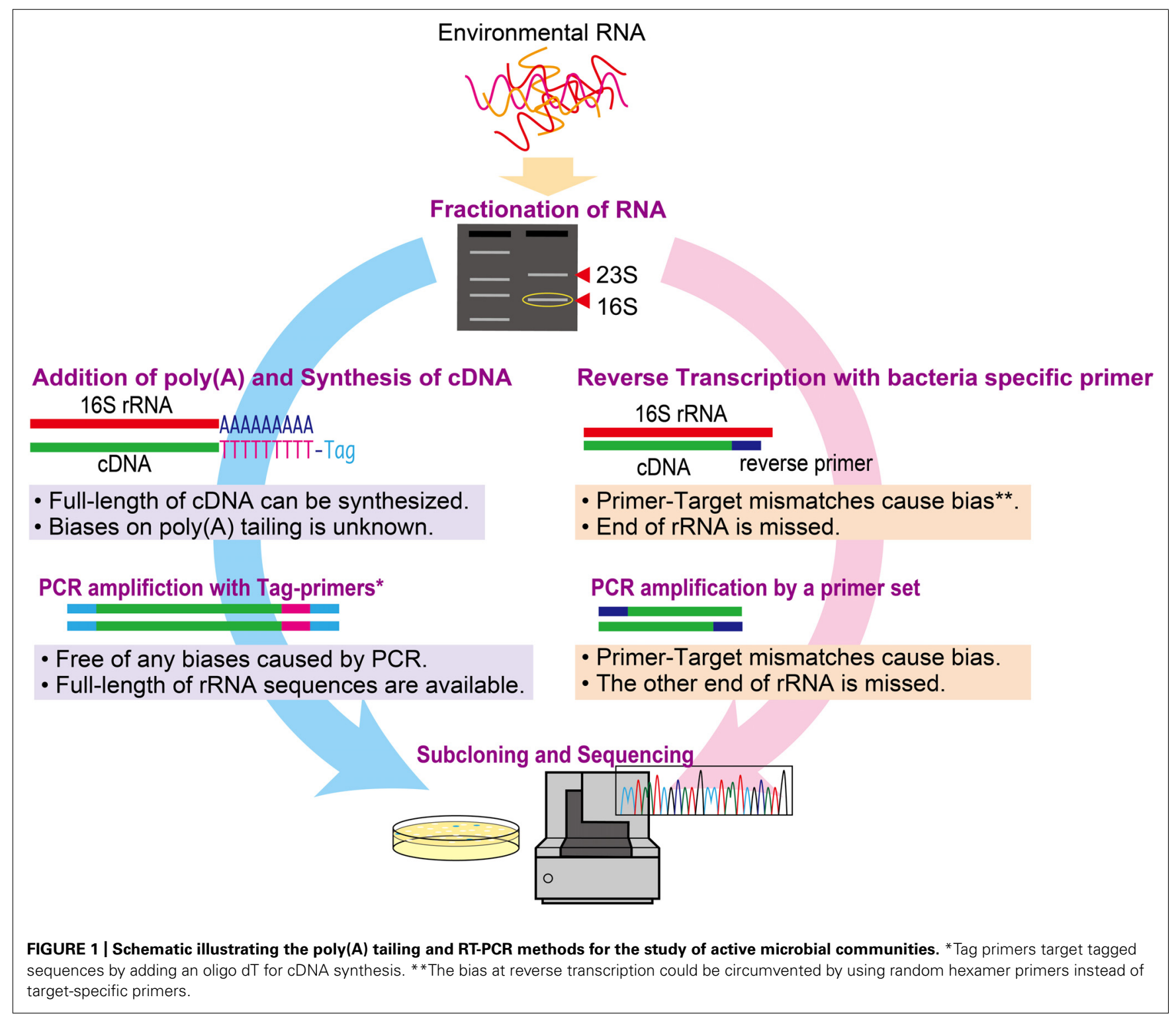

\section{DATA ANALYSIS}

Alignment of all 16S rRNA sequences was performed using the ARB software package (Ludwig et al., 2004). Since some of the $16 \mathrm{~S}$ rRNA sequences were fragmented after poly(A) tailing, a 600-bp fragment (corresponding to E. coli 16S rRNA positions 287-886) was used for comparisons of microbial diversity and community structure. Taxonomic assignments were made using Silva taxonomy and the Bayesian classifier. clustering sequences, calculation of diversity indices (i.e., Shannon and Simpson indices) and Libshuff test (Singleton et al., 2001; Schloss et al., 2004) were performed using the Mothur software package (Schloss et al., 2009; Hoshino et al., 2011). Phylogenetic tree was constructed by ARB software (Ludwig et al., 2004) using the neighbor-joining method (Saitou and Nei, 1987) with an Olsen correction. The coverage rate of the used primer set (26F-1492R) at the genus level was evaluated using TestPrime 1.0 program (Klindworth et al., 2013) using SILVA database SSU r114 with RefNR.

\section{NUCLEOTIDE SEQUENCE ACCESSION NUMBERS}

All 16S rRNA sequences obtained in this study were deposited in the DDBJ/EMBL/GenBank nucleotide sequence databases under the accession numbers KC470861-KC471309.

\section{RESULTS AND DISCUSSION} HMMER SCREENING OF 16S rRNA

HMMER screening of $16 \mathrm{~S}$ rRNA sequences obtained using the poly(A) tailing method resulted in the detection of 115 and 144 bacterial 16S rRNA sequences for the NEB and Takara poly(A) polymerase reactions, respectively. Approximately a half number of the total cDNA sequences (i.e., 107 and 92 sequences in the NEB and Takara cDNA libraries, respectively) were found to be $23 \mathrm{~S}$ rRNA fragments according to the HMMER analysis. Interestingly, few cDNAs from mRNA were detected. The fragmented 23S rRNA sequences were excluded from the downstream analysis. Some fragmented 16S rRNA sequences were also 
observed in the poly $(\mathrm{A})$ tailing libraries, suggesting that part of the $16 \mathrm{~S}$ rRNA pool was damaged during the fractionation step by excision of the band and extraction of $16 \mathrm{~S}$ rRNA from aga rose gel.

Only one and three of the 16S rRNA sequences obtained using the NEB and Takara polymerases, respectively, were identified as archaeal 16S rRNA. This result was consistent with results from previous analyses of samples from the same location, which indicated that the archaeal population is generally smaller than the bacterial population (Yanagawa et al., 2012). In addition, a previous study of geothermally heated soil from Yellowstone National Park in the United States recovered no archaeal RNA sequences using the poly(A) tailing method, despite the fact that numerous archaeal 16S rRNA sequences were obtained using the PCR-based clone library method (Botero et al., 2005). Therefore, poly(A) tailing might have bias which underestimate archaeal population although it is unknown whether the low abundance of archaeal sequences in the poly (A) tailing libraries is from the native archaeal abundance or due to this bias. It is important to note here that RNA-based methods depend on the recovery of intracellular RNA; therefore, the results cannot be correlated with the cellular biomass or DNA copy number of the genomic pool.

\section{COMPARISON OF MICROBIAL DIVERSITY}

More than $85 \%$ of the total $16 \mathrm{~S}$ rRNA sequences obtained using the poly(A) tailing and conventional RT-PCR methods were found to be derived from the Deltaproteobacteria, indicating that sulfatereducing bacteria are predominant members of sedimentary habitats (Figure 2, pie charts on the left). Conventional RT-PCR analysis identified 94\% (169/179) of the sequences obtained as Deltaproteobacteria, whereas $85 \%(98 / 115)$ and $88 \%(127 / 144)$ of the bacterial 16S rRNA sequences obtained using the NEB and Takara polymerase poly(A) tailing methods, respectively, were identified as Deltaproteobacteria (Figure 2, pie charts on the left). Overall, these results are consistent with those of a previous RNA-based study of the same hydrothermal field (Yanagawa et al., 2012).

The Deltaproteobacteria orders Desulfuromonadales and Desulfobacterales, both of which contain sulfur- and/or sulfatereducing bacteria, consistently appeared as predominant phylotypes in the clone libraries. However, there was a clear difference in the clonal frequency between libraries constructed using the two methods; the RT-PCR method indicated the predominance of Desulfuromonadales, while the poly(A)-tailing method indicated that Desulfobacterales predominate (Figure 2).

The detected sequences affiliated with Desulfuromonadales were mainly composed by the genera Pelobacter, Geoalkalibacter, and Geopsychrobacter (Figure 3). Almost half of the sequences from RT-PCR (84/179) and more than 25 sequences from both poly(A) libraries were classified to be Pelobacter, indicating predominance of this genus in the environment. TestPrime analysis (Klindworth etal., 2013) indicated that the coverage rates of the $26 \mathrm{~F}$ and $1492 \mathrm{R}$ primers with perfect match for Pelobacter, Geoalkalibacter, and Geopsychrobacter are 50.0, 75.0, and 100\%, respectively. On the other hand, the detected sequences of Desulfobacterales mainly consist of the genera Desulfopila, Desulfofaba, and Desulforhopalus (Figure 3), for which the coverage rates are 40,
100 , and 41.7\%, respectively. Among those three genera, the Desulfopila-related sequences were predominant in both poly (A) tailing clone libraries. The coverage rates of the detected genera within the Desulfobacterales were lower than those of Desulfuromonadales, resulting in lower abundance of Desulfobacterales in the RT-PCR libraries. Therefore, we infer that primer-dependent RTPCR assay overestimated Desulfuromonadales but underestimated Desulfobacterales due to the primer bias.

Representatives of the Gammaproteobacteria and Sphingobacteria were relatively minor components of all three libraries we examined. Although some sequences derived from Lentisphaerae and Holophagae were only detected by RT-PCR, the poly (A) tailing libraries constructed using the two different polymerases revealed more diverse lineages than did the RT-PCR library. The clone libraries obtained from poly(A) tailing included some classes that were not detected by RT-PCR, such as Nitrospira, Alphaproteobacteria, and Caldilineae.

In theory, poly(A) tailing methods could also be used to obtain archaeal $16 \mathrm{~S}$ rRNA, although a previous study failed to retrieve any archaeal 16S rRNA from geothermally heated soils from Yellowstone National Park (Botero et al., 2005). In this study, a total of four archaeal $16 \mathrm{~S}$ rRNA sequences were obtained using the poly $(\mathrm{A})$ tailing method.

Two of these sequences were derived from Candidatus "Parvarchaeum" (Baker etal., 2010), which belonged to Deep-sea Hydrothermal Vent Euryarchaeotic Group (DHVEG-6; Takai and Horikoshi, 1999), while the other two sequences formed a new branch distinct from the ancient archaeal group (AAG; Takai and Horikoshi, 1999; Figure 4). Organisms belonging to DHVEG-6 are primarily associated with deep-sea hydrothermal vent systems (Takai and Horikoshi, 1999; Teske and Sørensen, 2008; Nunoura et al., 2012), but have also been found in marine sediment and anoxic soil. The AAG were first described as a hydrothermal vent lineage, and, consistent with the results of this study, were later found in the cold organic-rich subsurface environment (Sørensen and Teske, 2006). Due to primer mismatching, there have been few reports to date of the use of conventional PCR with published primer sets to detect the four archaeal 16S rRNA sequences we detected in this study. For example, all four sequences have one mismatch to A806F (Wang and Qian, 2009), while Arch958R (DeLong, 1992) has six mismatches to T_34 and N_100, and two mismatches to T_35 and T_36.

In addition, we retrieved $23 \mathrm{~S}$ rRNA by poly(A) tailing with Takara polymerase: a total of 78 partial $23 \mathrm{~S}$ rRNA sequences ( $\sim 600$ bp in length) were obtained. Although classification of the $23 \mathrm{~S}$ rRNA sequences might be insufficient for the genus-level classification due to the limited number of $23 \mathrm{~S}$ rRNA in the database, we found predominance of Deltaproteobacteria (60/78) containing Desulfobacterales (19/78), Desulfuromonadales (20/78), and unclassified sequences (19/78), consistently supporting our observation of $16 \mathrm{~S}$ rRNA gene sequences.

\section{COMPARISON OF MICROBIAL COMMUNITY STRUCTURES}

To compare the microbial community structures indicated by the poly $(\mathrm{A})$ tailing and RT-PCR approaches, we calculated Shannon $\left(H^{\prime}\right)$ and Simpson diversity $(1 / D)$ indices for the 16S rRNA libraries. The highest diversity value was for the poly(A)-tailed 


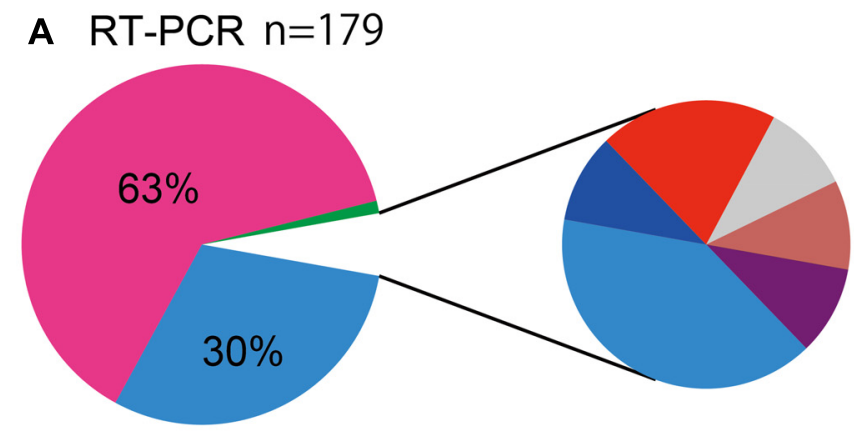

B NEB $n=115$

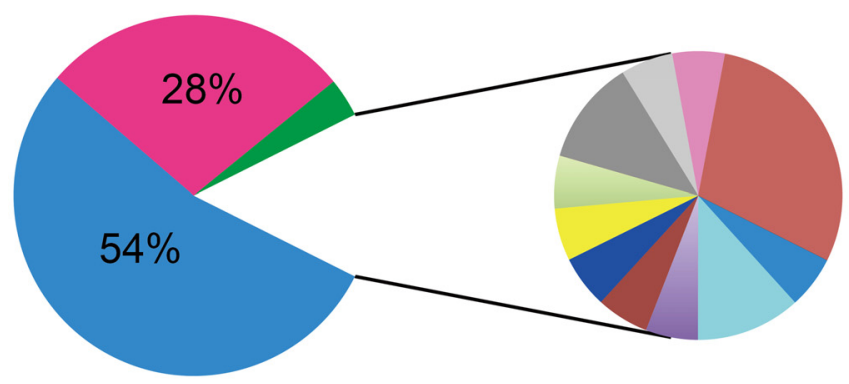

C TaKaRa $n=144$

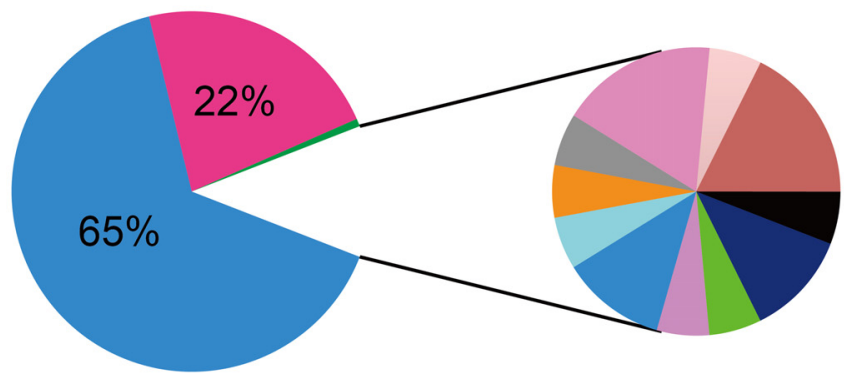

Desulfobacterales

Desulfuromonadales

Other deltaproteobacteria

Acidobacteria

Holophagae

Bacteroidia

Flavobacteria

Sphingobacteria

Caldilineae

GIF3

vadinBA26

SubsectionIII

Unclassified

Deferribacterales

Clostridia

Fusobacteria

Lentisphaeria

Nitrospira

Phycisphaerae

Alphaproteobacteria

Candidatus_Thiobios

Gammaproteobacteria

TA18

FIGURE 2 |The community structure of marine sediment microbial populations inferred from the results of RT-PCR and poly(A) tailing analyses. Deltaproteobacteria (left) and other bacterial populations (right) were shown in pie charts. Chart (A) indicated microbial community obtained by RT-PCR, whereas chart (B) and chart (C) were by poly(A) tailing with NEB and Takara poly(A) polymerase, respectively.

sequences obtained using the NEB polymerase (Table 1). For the unique poly(A)-tailed sequences (i.e., singletons), the highest diversity indices were obtained using the Takara polymerase. In contrast, the RT-PCR method was associated with the lowest diversity indices, regardless of the similarity cutoff used or not (Table 1). The results of Libshuff analysis indicate that the two poly(A) clone libraries are statistically different from that of RT-PCR whereas poly(A) libraries are not significantly different (Table 2). Overall, these results indicate that the poly(A) tailing methods retrieve more diverse $16 \mathrm{~S}$ rRNA sequences from the environment than does the conventional RT-PCR approach. In other words, it is important to recognize that primer-dependent molecular ecological approaches carry a risk of bias that could result in underestimation of microbial diversity. The bias effect may be more significant for microbial communities in rare and/or extreme habitats that have never been explored because we do not know exactly what organisms reside there.

\section{CONCLUSION AND PERSPECTIVES}

For decades, PCR-mediated molecular ecological approaches have been used to investigate the diversity of microbial communities in a variety of natural habitats. The primer sequences for amplifying $16 \mathrm{~S}$ rRNA (or its gene fragments) are based on known sequences contained in databases, targeting conserved regions that cover specific taxonomic groups. In this context, a critical issue in microbial ecology is the possibility of bias caused by mismatches between the published primers and the target sequences, especially for unidentified constituents of microbial communities in natural habitats. Bias of this sort has caused significant differences in estimates of microbial diversity and community structure, and also increases the difficulty of detecting previously unidentified organisms in the environment.

The poly(A) tailing of environmental $16 \mathrm{~S}$ rRNA is totally independent of published PCR primers. In this study, we clearly showed that the poly(A) tailing approach holds potential for 


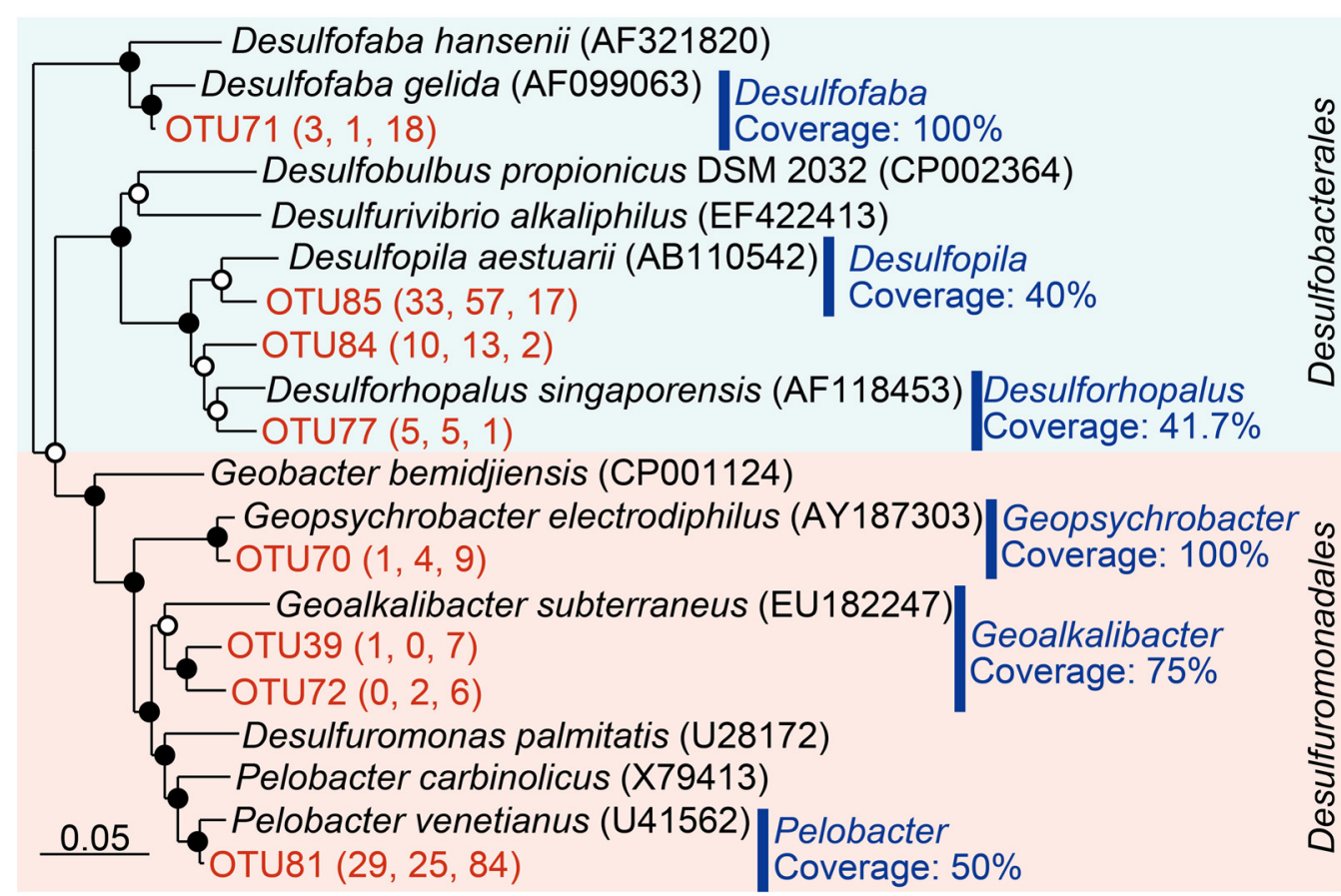

FIGURE 3 | Phylogenetic classification of Deltaproteobacteria sequences obtained in this study. The tree was constructed by neighbor-joining analysis with an Olsen correction. Operational taxonomic units (OTUs) were defined as the clusters at $97 \%$ sequence identity and only OTUs containing more than five sequences were shown in the tree. The numbers in parenthesis indicate the number of clones obtained by NEB poly $(\mathrm{A})$ polymerase, Takara poly (A) polymerase, and conventional RT-PCR, respectively (from left to right). Coverage of the primer set (26F-1492R) determined by TestPrime (http://www.arb-silva.de/search/testprime/) is shown for each genus. Bootstrap values are shown at branch nodes by closed circles $(>80 \%)$ and open circles $(<80 \%)$ as percentages of 1,000 replicates. Scale bar indicates $5 \%$ sequence divergence.

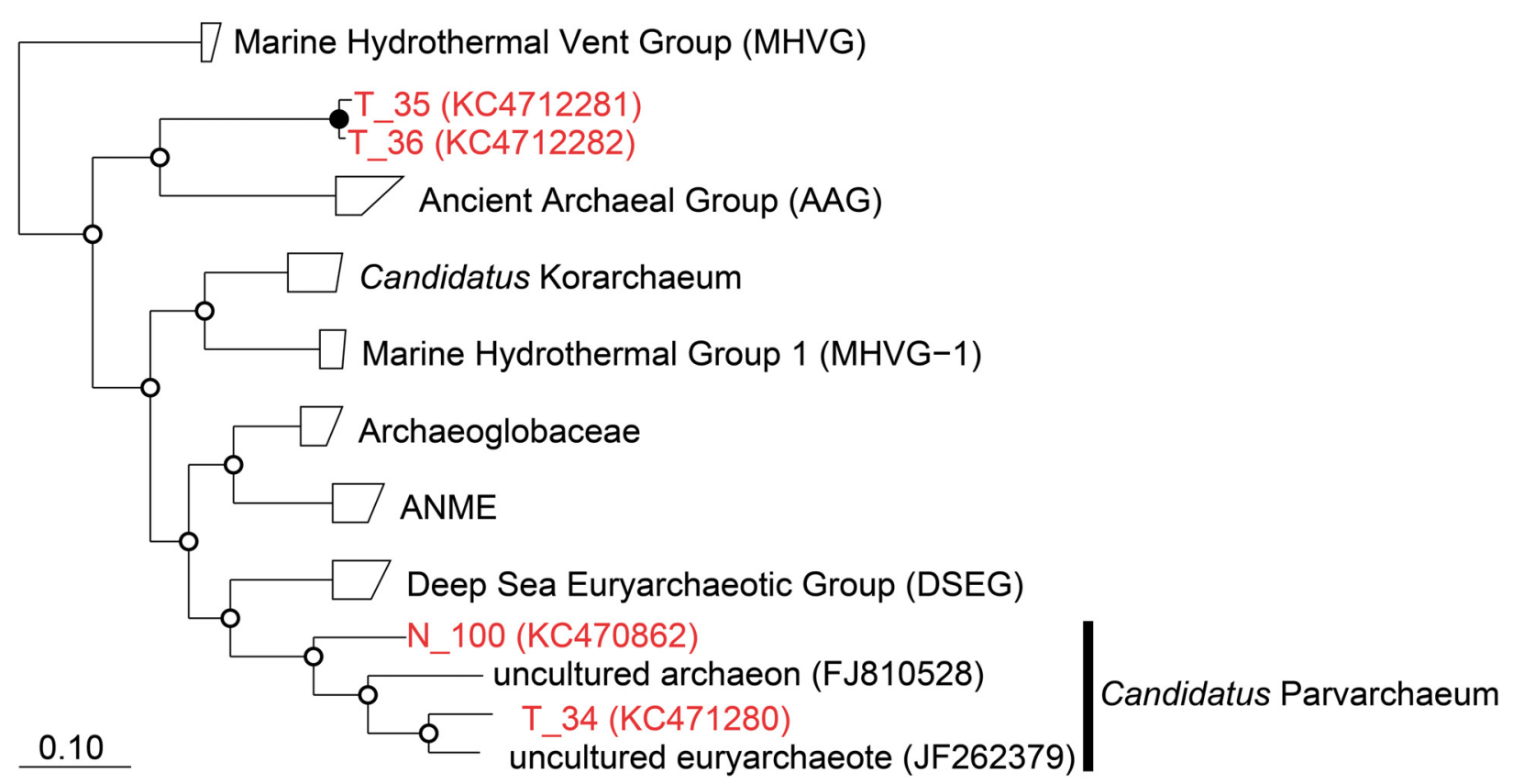

FIGURE 4 | Phylogenetic classification of archaeal 16S rRNA sequences obtained in this study. The tree was constructed by neighbor-joining analysis with an Olsen correction. Bootstrap values were shown at branch point as percentages of 1,000 replicates. Bootstrap values are shown at branch nodes by closed circles ( $>80 \%$ ) and open circles $(<80 \%)$ as percentages of 1,000 replicates. Scale bar indicated $10 \%$ sequence divergence. 
Table 1 | Diversity indices.

\begin{tabular}{|c|c|c|c|c|c|c|}
\hline \multirow[t]{2}{*}{ Similarity cutoff (\%) } & \multicolumn{3}{|c|}{ Shannon diversity index $\left(H^{\prime}\right)$} & \multicolumn{3}{|c|}{ Simpson diversity index (1/D) } \\
\hline & RT-PCR & Poly(A) NEB & Poly(A) Takara & RT-PCR & Poly(A) NEB & Poly(A) Takara \\
\hline 0 (unique) & 4.41 & 4.22 & 4.54 & 49.17 & 64.90 & 70.52 \\
\hline 3 & 2.23 & 2.48 & 2.41 & 4.13 & 6.53 & 5.16 \\
\hline
\end{tabular}

NEB: New England BioLabs Escherichia coli poly(A) polymerase. Takara: Takara poly(A) polymerase.

Table $2 \mid \boldsymbol{P}$-values* estimating similarity among each treatment generated using Libshuff $(10,000$ randomizations) among the three clone libraries.

\begin{tabular}{llll}
\hline & \multicolumn{3}{c}{$\boldsymbol{P}$-value comparison of library $(\boldsymbol{Y})$ with $\boldsymbol{X a}$} \\
\cline { 2 - 4 } Library $(\boldsymbol{X})$ & RT-PCR & NEB poly(A) & Takara poly(A) \\
\hline RT-PCR & $/$ & $\mathbf{0 . 0 0 0 1}$ & $\mathbf{0 . 0 0 0 3}$ \\
NEB poly(A) & $<\mathbf{0 . 0 0 0 1}$ & $/$ & 0.4009 \\
Takara poly(A) & $\mathbf{0 . 0 0 5 1}$ & 0.2407 & $/$
\end{tabular}

* $P$-values comparing either $X$ to $Y$ or $Y$ to $X$ indicate that the two communities are significantly different bold face values indicated significant difference $(P<0.0085$, employing the Bonferroni correction).

understanding of naturally occurring active microbial communities. This approach also has great potential for facilitating the

\section{REFERENCES}

Acinas, S. G., Sarma-Rupavtarm, R., Klepac-Ceraj, V., and Polz, M. F. (2005). PCR-induced sequence artifacts and bias: insights from comparison of two 16S rRNA clone libraries constructed from the same sample. Appl. Environ. Microbiol. 71, 89668969. doi: 10.1128/AEM.71.12.89668969.2005

Amann, R. I., Binder, B. J., Olson, R. J., Chisholm, S. W., Devereux, R., and Stahl, D. A. (1990). Combination of 16S rRNA-targeted oligonucleotide probes with flow cytometry for analyzing mixed microbial populations. Appl. Environ. Microbiol. 56, 1919-1925.

Baker, B. J., Comolli, L. R., Dick, G. J., Hauser, L. J., Hyatt, D., Dill, B. D., et al. (2010). Enigmatic, ultrasmall, uncultivated archaea. Proc. Natl. Acad. Sci. U.S.A. 107, 88068811. doi: 10.1073/pnas.0914470107

Baker, B. J., Tyson, G. W., Webb, R. I., Flanagan, J., Hugenholtz, P., Allen, E. E., et al. (2006). Lineages of acidophilic archaea revealed by community genomic analysis. Science 314 , 1933-1935. doi: 10.1126/science. 1132690

Baker, G. C., Smith, J. J., and Cowan, D. A. (2003). Review and re-analysis of domain-specific $16 \mathrm{~S}$ primers. J. Microbiol. Methods 55, 541-555. doi: 10.1016/j.mimet.2003.08.009

Ben-Dov, E., Shapiro, O. H., and Kushmaro, A. (2012). 'Next-base' effect on PCR amplification. Environ. Microbiol. Rep. 4, 183-188. doi: 10.1111/j.1758-2229.2011.00318.x

Botero, L. M., D’Imperio, S., Burr, M., Mcdermott, T. R., Young, M., and Hassett, D. J. (2005). Poly(A) polymerase modification and reverse transcriptase PCR amplification of environmental RNA. Appl. Environ. Microbiol. 71, 1267-1275. doi: 10.1128/AEM.71.3.1267-1275. 2005

Bru, D., Martin-Laurent, F., and Philippot, L. (2008). Quantification of the detrimental effect of a single primer-template mismatch by real-time PCR using the $16 \mathrm{~S}$ rRNA gene as an example. Appl. Environ. Microbiol. 74, 1660-1663. doi: 10.1128/AEM.02403-07

DeLong, E. F. (1992). Archaea in coastal marine environments. Proc. Natl. Acad. Sci. U.S.A. 89, 5685-5689. doi: 10.1073/pnas.89.12.5685

Eddy, S. R. (1998). Profile hidden Markov models. Bioinformatics 14, 755-763. doi: 10.1093/bioinformatics/14.9.755 discovery of as yet unknown microbes for which their 16S rRNA gene sequence do not match published primer sequences, although the potential bias of poly(A) tailing to rRNA genes needs to be studied further. By combining this approach with "deep sequencing" NGS technologies that allow for sequencing full-length $16 \mathrm{~S}$ rRNAs, it may be possible in the future to obtain a detailed view of the true structure of microbial communities in natural habitats.

\section{ACKNOWLEDGMENTS}

The authors thank Ms. Sayo Hashimoto for technical assistance. This work was supported in part by the JSPS Strategic Fund for Strengthening Leading-edge Research and Development (to JAMSTEC), by a Sasakawa Scientific Research Grant from the Japan Science Society (to Tatsuhiko Hoshino), and by the JSPS Funding Program for Next Generation World-leading Researchers (NEXT Program, to Fumio Inagaki).

Edgar, R. C., Haas, B. J., Clemente, J. C., Quince, C., and Knight, R. (2011). UCHIME improves sensitivity and speed of chimera detection. Bioinformatics 27, 21942200. doi: 10.1093/bioinformatics/ btr381

Fuhrman, J. A. (2009). Microbial community structure and its functional implications. Nature 459, 193-199. doi: 10.1038/nature08058

Hicks, R. E., Amann, R. I., and Stahl, D. A. (1992). Dual staining of natural bacterioplankton with 4',6-diamidino-2-phenylindole and fluorescent oligonucleotide probes targeting kingdom-level 16S rRNA sequences. Appl. Environ. Microbiol. 58, 2158-2163.

Hong, S., Bunge, J., Leslin, C., Jeon, S., and Epstein, S. S. (2009). Polymerase chain reaction primers miss half of rRNA microbial diversity. ISME J. 3, 1365-1373. doi: 10.1038/ ismej.2009.89

Hoshino, T., Morono, Y., Terada, T., Imachi, H., Ferdelman, T. G., and Inagaki, F. (2011). Comparative study of subseafloor microbial community structures in deeply buried coral fossils and sediment matrices from the challenger mound in the porcupine seabight. Front. Microbiol.
2:231. doi: 10.3389/fmicb.2011. 00231

Huang, Y., Gilna, P., and Li, W. (2009). Identification of ribosomal RNA genes in metagenomic fragments. Bioinformatics 25, 1338-1340. doi: 10.1093/bioinformatics/btp161

Inagaki, F., Sakihama, Y., Inoue, A., Kato, C., and Horikoshi, K. (2002). Molecular phylogenetic analyses of reverse-transcribed bacterial rRNA obtained from deep-sea cold seep sediments. Environ. Microbiol. 4, 277-286. doi: 10.1046/j.14622920.2002.00294.x

Klindworth, A., Pruesse, E., Schweer, T., Peplies, J., Quast, C., Horn, M., et al. (2013). Evaluation of general $16 \mathrm{~S}$ ribosomal RNA gene PCR primers for classical and next-generation sequencing-based diversity studies. Nucleic Acids Res. 41, e1. doi: 10.1093/ nar/gks808

Lagesen, K., Hallin, P., Rodland, E. A., Staerfeldt, H. H., Rognes, T., and Ussery, D. W. (2007). RNAmmer: consistent and rapid annotation of ribosomal RNA genes. Nucleic Acids Res. 35, 3100-3108. doi: 10.1093/nar/gkm160

Liu, W. T., Marsh, T. L., Cheng, H., and Forney, L. J. (1997). Characterization of microbial diversity by determining 
terminal restriction fragment length polymorphisms of genes encoding $16 \mathrm{~S}$ rRNA. Appl. Environ. Microbiol. 63, 4516-4522.

Loy, A., Lehner, A., Lee, N., Adamczyk, J., Meier, H., Ernst, J., et al. (2002). Oligonucleotide microarray for $16 \mathrm{~S}$ rRNA gene-based detection of all recognized lineages of sulfate-reducing prokaryotes in the environment. Appl. Environ. Microbiol. 68, 50645081. doi: 10.1128/AEM.68.10.50645081.2002

Ludwig, W., Strunk, O., Westram, R., Richter, L., Meier, H., Yadhukumar, etal. (2004). ARB: a software environment for sequence data. Nucleic Acids Res. 32, 1363-1371. doi: 10.1093/nar/gkh293

Nunoura, T., Takaki, Y., Kazama, H., Hirai, M., Ashi, J., Imachi, H., et al. (2012). Microbial diversity in deep-sea methane seep sediments presented by SSU rRNA gene tag sequencing. Microbes Environ. 27, 382-390. doi: 10.1264/jsme2. ME12032

Raynal, L. C., and Carpousis, A. J. (1999). Poly(A) polymerase I of Escherichia coli: characterization of the catalytic domain, an RNA binding site and regions for the interaction with proteins involved in mRNA degradation. Mol. Microbiol. 32, 765-775. doi: 10.1046/j.13652958.1999.01394.x

Saitou, N., and Nei, M. (1987). The neighbor-joining method: a new method for reconstructing phylogenetic trees. Mol. Biol. Evol. 4, 406-425.

Schloss, P. D., Larget, B. R., and Handelsman, J. (2004). Integration of microbial ecology and statistics: a test to compare gene libraries. Appl. Environ. Microbiol. 70, 5485-5492. doi: 10.1128/AEM.70.9.5485-5492. 2004
Schloss, P. D., Westcott, S. L., Ryabin, T., Hall, J. R., Hartmann, M., Hollister, E. B., et al. (2009). Introducing mothur: open-source, platform-independent, communitysupported software for describing and comparing microbial communities. Appl. Environ. Microbiol. 75, 7537-7541. doi: 10.1128/AEM. 01541-09

Sillero, M. A., Socorro, S., Baptista, M. J., Del Valle, M., De Diego, A., and Sillero, A. (2001). Poly(A) polymerase from Escherichia coli adenylylates the $3^{\prime}$-hydroxyl residue of nucleosides, nucleoside $5^{\prime}$-phosphates and nucleoside $\left(5^{\prime}\right)$ oligophospho $\left(5^{\prime}\right)$ nucleosides $(\mathrm{NpnN})$. Eur. J. Biochem. 268, 3605-3611. doi: 10.1046/j.1432-1327.2001.02271.x

Singleton, D. R., Furlong, M. A., Rathbun, S. L., and Whitman, W. B. (2001). Quantitative comparisons of $16 \mathrm{~S}$ rRNA gene sequence libraries from environmental samples. Appl. Environ. Microbiol. 67, 43744376. doi: 10.1128/AEM.67.9.43744376.2001

Sipos, R., Szekely, A. J., Palatinszky, M., Revesz, S., Marialigeti, K., and Nikolausz, M. (2007). Effect of primer mismatch, annealing temperature and PCR cycle number on 16S rRNA genetargetting bacterial community analysis. FEMS Microbiol. Ecol. 60, 341350. doi: 10.1111/j.1574-6941.2007. 00283.x

Sogin, M. L., Morrison, H. G., Huber, J. A., Welch, D. M., Huse, S. M., Neal, P. R., et al. (2006). Microbial diversity in the deep sea and the underexplored "rare biosphere". Proc. Natl. Acad. Sci. U.S.A. 103, 12115-12120. doi: 10.1073/pnas.0605127103

Sørensen, K. B., and Teske, A. (2006). Stratified communities of active Archaea in deep marine subsurface sediments. Appl. Environ. Microbiol. 72, 4596-4603. doi: 10.1128/ AEM.00562-06

Takai, K., and Horikoshi, K. (1999). Genetic diversity of archaea in deepsea hydrothermal vent environments. Genetics 152, 1285-1297.

Takami, H., Noguchi, H., Takaki, Y., Uchiyama, I., Toyoda, A., Nishi, S. etal. (2012). A deeply branching thermophilic bacterium with an ancient acetyl-CoA pathway dominates a subsurface ecosystem. PLoS ONE 7:e30559. doi: 10.1371/journal.pone.0030559

Teske, A., and Sørensen, K. B. (2008). Uncultured archaea in deep marine subsurface sediments: have we caught them all? ISME J. 2, 3-18.

Vergin, K. L., Urbach, E., Stein, J. L., Delong, E. F., Lanoil, B. D., and Giovannoni, S. J. (1998). Screening of a fosmid library of marine environmental genomic DNA fragments reveals four clones related to members of the order Planctomycetales. Appl. Environ. Microbiol. 64, 3075-3078.

Walters, W. A., Caporaso, J. G., Lauber C. L., Berg-Lyons, D., Fierer, N., and Knight, R. (2011). PrimerProspector: de novo design and taxonomic analysis of barcoded polymerase chain reaction primers. Bioinformatics 27 1159-1161. doi: 10.1093/bioinformatics/btr087

Wang, Y., and Qian, P. Y. (2009). Conservative fragments in bacterial 16S rRNA genes and primer design for $16 \mathrm{~S}$ ribosomal DNA amplicons in metagenomic studies. PLoS ONE 4:e7401. doi: 10.1371/journal.pone.0007401

Webster, N. S., Taylor, M. W., Behnam, F., Lucker, S., Rattei, T., Whalan, S., et al. (2010). Deep sequencing reveals exceptional diversity and modes of transmission for bacterial sponge symbionts. Environ. Microbiol. 12, 2070-2082.

Yanagawa, K., Morono, Y., De Beer, D., Haeckel, M., Sunamura, M., Futagami, T., et al. (2012). Metabolically active microbial communities in marine sediment under high-CO2 and low-pH extremes. ISME J. 7, 555-567. doi: 10.1038/ismej.2012.124

Zheng, D., Alm, E. W., Stahl, D. A., and Raskin, L. (1996). Characterization of universal small-subunit rRNA hybridization probes for quantitative molecular microbial ecology studies. Appl. Environ. Microbiol. 62, 45044513.

Conflict of Interest Statement: The authors declare that the research was conducted in the absence of any commercial or financial relationships that could be construed as a potential conflict of interest.

Received: 05 March 2013; accepted: 31 May 2013; published online: 18 June 2013.

Citation: Hoshino $T$ and Inagaki $F$ (2013) A comparative study of microbial diversity and community structure in marine sediments using poly $(A)$ tailing and reverse transcription-PCR. Front. Microbiol. 4:160. doi: 10.3389/ fmicb.2013.00160

This article was submitted to Frontiers in Extreme Microbiology, a specialty of Frontiers in Microbiology.

Copyright (c) 2013 Hoshino and Inagaki. This is an open-access article distributed under the terms of the Creative Commons Attribution License, which permits use, distribution and reproduction in other forums, provided the original authors and source are credited and subject to any copyright notices concerning any thirdparty graphics etc. 